\title{
The impact of oxygen on the final alcohol content of wine fermented by a mixed starter culture
}

\author{
Pilar Morales • Virginia Rojas • Manuel Quirós • \\ Ramon Gonzalez
}

Received: 23 October 2014 / Revised: 4 December 2014 / Accepted: 10 December 2014 / Published online: 13 January 2015

(C) The Author(s) 2015. This article is published with open access at Springerlink.com

\begin{abstract}
We have developed a wine fermentation procedure that takes advantage of the metabolic features of a previously characterized Metschnikowia pulcherrima strain in order to reduce ethanol production. It involves the use of M. pulcherrima/Saccharomyces cerevisiae mixed cultures, controlled oxygenation conditions during the first $48 \mathrm{~h}$ of fermentation, and anaerobic conditions thereafter. The influence of different oxygenation regimes and initial inoculum composition on yeast physiology and final ethanol content was studied. The impact of oxygenation on yeast physiology goes beyond the first aerated step and influences yields and survival rates during the anaerobic stage. The activity of M. pulcherrima in mixed oxygenated cultures resulted in a clear reduction in ethanol yield, as compared to $S$. cerevisiae. Despite relatively low initial cell numbers, $S$. cerevisiae always predominated in mixed cultures by the end of the fermentation process. Strain replacement was faster under low oxygenation levels. M. pulcherrima confers an additional advantage in terms of dissolved oxygen, which drops to zero after a few hours of culture, even under highly aerated conditions, and this holds true for mixed cultures. Alcohol reduction
\end{abstract}

Electronic supplementary material The online version of this article (doi:10.1007/s00253-014-6321-3) contains supplementary material, which is available to authorized users.

P. Morales $(\varangle) \cdot$ V. Rojas $\cdot$ M. Quirós $\cdot$ R. Gonzalez $(\bowtie)$ Instituto de Ciencias de la Vid y del Vino (CSIC, Universidad de la Rioja, Gobierno de La Rioja), Logroño, La Rioja, Spain e-mail: pilar.morales@icvv.es

e-mail: ramon.gonzalez@csic.es

\section{Rojas}

Facultad de Ciencias Químicas, Universidad de Autónoma de San Luis Potosí, Centro Histórico, Mexico

Present Address:

M. Quirós

Evolva Biotech A/S, Copenhagen Ø, Denmark values about $3.7 \%(v / v)$ were obtained for mixed cultures under high aeration, but they were associated to unacceptable volatile acidity levels. In contrast, under optimized conditions, only $0.35 \mathrm{~g} / \mathrm{L}$ acetic acid was produced, for an alcohol reduction of $2.2 \%(v / v)$, and almost null dissolved oxygen during the process.

Keywords Low alcohol · Non-Saccharomyces yeast . Respiratory quotient · Dissolved oxygen · Volatile acidity

\section{Introduction}

Consumer preferences toward well-structured, full body wines have driven the requirement for late harvests in order to ensure an optimal phenolic maturity of grapes. In the context of global warming, this practice results in a noticeable increase in the sugar content of the berries at harvest (Mira de Orduña 2010) that finally gives rise to higher alcohol levels in wine. This leads to numerous wine quality, marketing, and public health issues. In order to compensate for this increase, the wine industry has been seeking for new approaches to reduce the alcohol content of wines. Several technological solutions including winemaking practices adapted to unripe berries (Kontoudakis et al. 2011; Canals et al. 2008) or partial dealcoholization by physical methods (Schmidtke et al. 2012; Catarino and Mendes 2011; Belisario-Sánchez et al. 2009) have been proposed to this end. Additionally, several research articles have focused on unconventional microbiological solutions for this purpose. Among them, the development of low alcohol yield yeast strains has been, and still is, a hot topic in the field of winemaking. In this context, different metabolic or evolutionary engineering strategies aiming to divert the carbon flux from ethanol production in Saccharomyces cerevisiae have been proposed (Michnick et al. 1997; Cadiere et al. 2011; Heux et al. 2006; Rossouw et al. 2013; 
Varela et al. 2012a). The topic has been extensively reviewed by Kutyna et al. (2010). However, modifying ethanol yields in this species has been proven to be a difficult task, mainly due to the tight regulation of the pyruvate node under anaerobic conditions (Varela et al. 2004; Quirós et al. 2013). Consequently, limited success has been generally achieved. Additionally, the industrial application of most of these approaches is currently limited by the concomitant overproduction of non-desired metabolites such as acetate, acetaldehyde or acetoin (Heux et al. 2006), public attitudes toward genetically modified organisms (GMOs) and/or regulations that restrict their usefulness in the wine industry. Recent reports indicate that experimental evolution might be a feasible alternative to genetic engineering in order to develop $S$. cerevisiae yeast strains with reduced alcohol yield (Tilloy et al. 2014).

While $S$. cerevisiae is the main yeast species responsible for conducting the alcoholic fermentation of grape must, the contribution of a non-negligible number of other yeast species to the initial stages of the process and to the sensorial properties of wine is currently well established (Fleet 2003; Medina et al. 2013; Ciani et al. 2010; Rojas et al. 2003; Sadoudi et al. 2012; Cordero-Bueso et al. 2013). These species, naturally present in sound grapes, are mainly represented by strains belonging to the apiculate yeast genus Hanseniaspora (mainly Hanseniaspora uvarum or its anamorph Kloeckera apiculata) and other species of the genera Candida, Pichia, Kluyveromyces, and Metschnikowia (Fleet 2007; Tamang and Fleet 2009).

Our research group recently proposed the possibility of using non-Saccharomyces yeast species for the reduction of the alcohol content of wine (Gonzalez et al. 2013). Key differences in sugar metabolism between some of these species and $S$. cerevisiae could actually allow for an increased breakdown of sugars via respiratory pathways rather than through fermentation, provided that an appropriate amount of oxygen is available. The possibility of using respiratory catabolism as a clean way to limit sugar conversion to ethanol had been previously suggested by other authors (Smith 1995; Erten and Campbell 2001; Bärwald and Fischer 1996). However, most of these studies describe preliminary results and went almost unnoticed for several reasons, including limited availability of the original documents (Smith 1995), limitations in the experimental setup, or low number of yeast strains screened. A Crabtree-negative recombinant $S$. cerevisiae wine yeast strain derivative developed by Henricsson et al. (2005) would also be interesting in this context. However, commercial application of such strain would experience the inconveniences associated to its GMO status.

In a recent work, our group surveyed around 60 nonSaccharomyces yeast strains of 29 different species to evaluate their potential application as starter cultures for lowering the ethanol content of wines (Quirós et al. 2014). This study, which set the focus on yeast key physiological parameters during the aerobic metabolism of synthetic must, concluded that high acetic acid yields constituted the main handicap for this application (Quirós et al. 2014). It was also found that high dissolved oxygen (DO) levels were not required for relevant yeast respiration (Quirós et al. 2014). Among the yeast species studied, different isolates belonging to the species Metschnikowia pulcherrima stood out due to their low ethanol and acetate yields on sugar and high sugar conversion rate. The usefulness of this non-conventional species for the aforementioned purpose was also remarked by Contreras et al. (2014), where a reduction in the alcohol level between 0.9 and $1.6 \%(v / v)$ was achieved in fermentations performed by sequential inoculation. Nevertheless, the potential contribution of respiration to alcohol reduction was not explored or discussed in that study.

As a proof of concept, we addressed the effect of different aeration conditions and different co-inoculation ratios of $S$. cerevisiae and M. pulcherrima strains selected from our previous work (Quirós et al. 2014), with the aim of achieving a significant reduction of the alcohol level of wine, while limiting volatile acidity production and the contact of grape must components with molecular oxygen.

\section{Materials and methods}

\section{Yeast strains}

A commercial S. cerevisiae wine yeast strain, EC1118 (Lallemand Inc., Montreal, Canada), and M. pulcherrima CECT12841, selected from a previous study (Quirós et al. 2014). The strains were grown at $28^{\circ} \mathrm{C}$ and maintained at $4{ }^{\circ} \mathrm{C}$ on yeast peptone dextrose (YPD) plates ( $2 \%$ glucose, $2 \%$ peptone, $1 \%$ yeast extract, and $2 \%$ agar), as well as in glycerol stocks at $-80{ }^{\circ} \mathrm{C}$.

\section{Controlled aeration fermentation assays}

Fermentation experiments were performed in triplicate (for cultures sparged with pure air or nitrogen) or duplicate (for intermediate aeration conditions), using MiniBio bioreactors ( $250 \mathrm{~mL}$ nominal volume) equipped with Peltier-refrigerated gas condensers (Applikon Biotechnology B.V., Delft, The Netherlands). Seed cultures were grown in YPD broth for $48 \mathrm{~h}$, at $25^{\circ} \mathrm{C}$ and $250 \mathrm{rpm}$. Bioreactors were filled with $150 \mathrm{~mL}$ of a filter-sterilized natural white grape must, a mixture of Malvasia and Viura varieties with no added carbon or nitrogen sources (262-265 g/L of sugars; $200 \mathrm{mg} / \mathrm{L}$ yeast assimilable nitrogen), and $200 \mu \mathrm{L}$ (approx.) of antifoam 204 (Sigma-Aldrich, St. Louis, MO). The same batch of grape must was used for all experiments. Temperature was set to 
$25{ }^{\circ} \mathrm{C}$, initial stirring to $1000 \mathrm{rpm}$, and inoculation to approximately 0.2 initial optical density at $600 \mathrm{~nm}\left(\mathrm{OD}_{600}\right)$. Initial proportions of both strains in co-inoculation experiments were based on $\mathrm{OD}_{600}$ values. The cultures were sparged in a discontinuous regime with pure $\mathrm{N}_{2}$, pure air, or mixtures of both, at a gas flow rate of $3.0 \mathrm{~L} / \mathrm{h}$ (i.e. 20 gas volumes/culture volume/h (vvh)). Gas flow was controlled with MFC17 mass flow controllers (Aalborg Instruments and Controls, Inc., Orangeburg, NY), whose calibration was regularly verified with a soap bubble flow meter. In a preliminary assay, aeration was automatically controlled from time zero in order to maintain a DO level above $15 \%$ (with air) in the pure culture of M. pulcherrima CECT12841. Sparging was totally interrupted $48 \mathrm{~h}$ after inoculation, and stirring slowed down to $200 \mathrm{rpm}$. Independently of the gas used and in order to improve reproducibility of the experiments, as well as to standardize any possible effect on the loss of volatile compounds, successive experiments were performed using a programmed on/off pattern for the gas valve, mimicking the pattern obtained in that preliminary experiment (Table S1 in the Supplementary Material). Samples for determination of metabolite concentrations were withdrawn every $12 \mathrm{~h}$ for the first 2 days and every $24 \mathrm{~h}$ thereafter. Population dynamics was monitored every $24 \mathrm{~h}$.

Viable counts in pure and mixed cultures

Evolution of global biomass in all fermentation assays was monitored by daily determination of $\mathrm{OD}_{600}$. Viable cells for each of the strains were quantified by plating appropriate dilutions in YPD plates and incubating for $48-72 \mathrm{~h}$ at $25^{\circ} \mathrm{C}$. Colonies of $S$. cerevisiae EC1118 in mixed cultures were distinguished from those of $M$. pulcherrima CECT12841 by the development of a pink coloration by the latter.

\section{Determination of metabolite concentrations}

The concentration of glucose, fructose, glycerol, ethanol, and acetic acid was determined using a Surveyor Plus Liquid Chromatograph (Thermo Fisher Scientific, Waltham, MA) equipped with a refraction index and a photodiode array detector (Surveyor RI Plus and Surveyor PDA Plus, respectively) on a $300 \times 7.7 \mathrm{~mm}$ HyperREZ ${ }^{\mathrm{TM}} \mathrm{XP}$ Carbohydrate $\mathrm{H}+$ ( $8 \mu \mathrm{m}$ particle size) column and guard (Thermo Fisher Scientific). The column was maintained at $50{ }^{\circ} \mathrm{C}$, and $1.5 \mathrm{mM} \mathrm{H}_{2} \mathrm{SO}_{4}$ was used as the mobile phase at a flow rate of $0.6 \mathrm{~mL} / \mathrm{min}$. Prior to injection in duplicate, the samples were filtered through $0.22 \mu \mathrm{m}$ pore size nylon filters (Micron Analitica, Madrid, Spain) and diluted 10-fold in MilliQ water.

Yeast assimilable nitrogen in natural grape must was determined spectrophotometrically as the sum of the contributions of free ammonium and free amino groups. Ammonium was assayed using a specific R-Biopharm assay kit (Darmstadt,
Germany). Free amino groups were determined with $o$ phthaldialdehyde (Dukes and Butzke 1998).

Statistical analysis

One way analysis of variance was carried out on the main fermentation metabolites found on day 2 and on finished fermentations sparged with pure air or nitrogen, with inoculum composition as main effect on each aeration condition. The effect of aeration on the main fermentation metabolites was also analyzed by means of one-way ANOVA on each inoculum composition. Means were compared using Tukey's test, with significance level set at $5 \%$. Data from fermentations sparged with gas mixtures $(10 \%$ or $25 \%$ air) were compared by Student's $t$ test with significance level set at $5 \%$. Correlation between main yields and air content in the sparging gas was analyzed by Pearson correlation analysis. All analyses were performed using SPSS Statistics v. 20 program (IBM, Armonk, NY).

\section{Results}

To establish the aeration regime for all further experiments in this study, we performed an initial assay with $M$. pulcherrima CECT12841 in natural grape must. The gas valve control was set to open every time DO level fell below $15 \%$ (with air) during the first $48 \mathrm{~h}$. This saturation level was taken as a suitable balance between the precision of oxygen saturation measurement and the expected yeast requirements for respiration. According to data from Saa et al. (2012) for oxygen solubility in fermentation media at $25{ }^{\circ} \mathrm{C}$ (i.e. about $7 \mathrm{mg} / \mathrm{L}$ ), $15 \%$ oxygen saturation (with air) would be equivalent to around $1 \mathrm{mg} / \mathrm{L}$ of molecular oxygen. The records of the experiment indicated the total opening time for the air valve that was about $30 \mathrm{~h}$. This information, as well as the timing of the actuator status (on/off), was used to design the gassing pattern employed in the subsequent experiments (Table S1 in the Supplementary Material). In order to minimize any ambiguity in the interpretation of the analytical results that could have arisen from variations in the gassing regimes (mainly concerning ethanol stripping), all the experiments analyzed in this work were sparged following the above-mentioned pattern and with exactly the same gas flow (20 vvh), independently of the composition of the sparging gas.

We had previously demonstrated respiratory metabolism for M. pulcherrima CECT12841, under similar conditions as described in "Materials and methods" with lower but continuous air flow $(1.2 \mathrm{~L} / \mathrm{h})$. Indeed respiratory quotient (RQ) values remained close to 1 (indicating pure respiration) until dissolved oxygen levels were undetectable (Quirós et al. 
2014). From that point, a steady increase in RQ values was observed. S. cerevisiae EC1118 also showed respirofermentative metabolism under such conditions but always with higher RQ values than M. pulcherrima (Quirós et al. 2014).

Impact of M. pulcherrima CECT12841 and aeration on the initial yield of the main fermentation metabolites

Initial assays were performed using either air or nitrogen as sparging gas and four different strain combinations: pure cultures of $S$. cerevisiae EC1118 or M. pulcherrima CECT12841, and mixed cultures with the same inoculation level of $M$. pulcherrima, plus 1 or $10 \%$ the inoculation level of $S$. cerevisiae used in the pure culture.

Concentration and yield of the main fermentation metabolites during the aerated step (first $48 \mathrm{~h}$ of culture) are shown in Table 1. For $S$. cerevisiae, a clear impact of aeration is observed on the yield of glycerol, ethanol, and acetic acid. Glycerol and ethanol yields decreased with air, while acetic acid yield increased by more than five times (Table 1).

M. pulcherrima responded differently to aeration (Table 1). On one side, sugar consumption clearly increased with aeration. On the other side, ethanol yield decreased to a higher extent than observed for S. cerevisiae (from 0.357 to $0.197 \mathrm{~g}$ / g). Finally, under air sparging conditions, M. pulcherrima cultures showed a lower acetic acid yield and higher glycerol yield than $S$. cerevisiae (Table 1).
Mixed cultures showed (i) lower sugar consumption values than those of $S$. cerevisiae under both aerobic and anaerobic conditions; (ii) similar acetic acid yields to those of M. pulcherrima, with little impact of oxygen availability (resulting in values clearly lower than $S$. cerevisiae under these conditions); and (iii) a large reduction in ethanol yield when sparged with air, down to values about half those of S. cerevisiae (Table 1). Up to this point, no statistically significant differences were found between cultures inoculated with 1 or $10 \%$ S. cerevisiae (Table 1).

Long-term impact of initial aeration

After 11 days, sugars were totally consumed, both for S. cerevisiae and mixed cultures. However, fermentations with pure $M$. pulcherrima cultures were sluggish, with more than $50 \mathrm{~g} / \mathrm{L}$ residual sugar at this time point (data not shown).

By the end of the fermentation, a clear increase in ethanol yield was observed for all cultures (Table 2), as compared to data for the first 2 days.

Stage-specific yields on glucose for the non-aerated stage were calculated taking into account the sugar consumed and the increase in ethanol from day 2 to the end of fermentation. Ethanol yields on glucose for this stage ranged from 0.431 to $0.481 \mathrm{~g} / \mathrm{g}$ (Table 3). Different stage-specific yields were also observed for acetic acid and glycerol as a function of both inoculum and sparging gas composition (Table 3).

Table 1 Concentration and yields of the main fermentation metabolites for fermentations sparged with air or nitrogen, in the conditions described in the text, after $48 \mathrm{~h}$ of culture (end of the aerated step

\begin{tabular}{|c|c|c|c|c|c|}
\hline & & S. cerevisiae ${ }^{\mathrm{a}}$ & $\begin{array}{l}\text { M. pulcherrima } \\
+10 \% \text { S. } \text { cerevisiae }^{\mathrm{a}}\end{array}$ & $\begin{array}{l}\text { M. pulcherrima } \\
+1 \% \text {. cerevisiae }\end{array}$ & M. pulcherrima ${ }^{\mathrm{a}}$ \\
\hline \multirow[t]{2}{*}{ Glycerol $(\% w / v)$} & $\operatorname{Air}^{\mathrm{b}}$ & $0.69 \pm 0.03 * \mathrm{~A}$ & $1.23 \pm 0.15 \mathrm{~B}$ & $1.21 \pm 0.04 * \mathrm{~B}$ & $1.18 \pm 0.17 \mathrm{~B}$ \\
\hline & Nitrogen $^{\mathrm{b}}$ & $0.93 \pm 0.08 *$ & $1.05 \pm 0.03$ & $1.09 \pm 0.06^{*}$ & $1.05 \pm 0.11$ \\
\hline \multirow[t]{2}{*}{ Ethanol $(\% v / v)$} & $\operatorname{Air}^{b}$ & $5.1 \pm 0.2 * \mathrm{~B}$ & $1.8 \pm 0.5 * \mathrm{~A}$ & $2.0 \pm 0.5 * \mathrm{~A}$ & $2.6 \pm 1.5 \mathrm{~A}$ \\
\hline & Nitrogen ${ }^{\mathrm{b}}$ & $6.7 \pm 0.5^{*} \mathrm{C}$ & $5.1 \pm 0.1 * \mathrm{~B}$ & $4.0 \pm 0.1 * \mathrm{~A}$ & $3.2 \pm 0.5 \mathrm{~A}$ \\
\hline \multirow[t]{2}{*}{ Acetic acid (mg/L) } & $\mathrm{Air}^{\mathrm{b}}$ & $878 \pm 62 * \mathrm{~B}$ & $93 \pm 43 \mathrm{~A}$ & $86 \pm 31 \mathrm{~A}$ & $83 \pm 25 \mathrm{~A}$ \\
\hline & Nitrogen & $165 \pm 125^{*}$ & $64 \pm 16$ & $58 \pm 5$ & $45 \pm 11$ \\
\hline \multirow[t]{2}{*}{ Cons. sugars $(\% w / v)$} & $\operatorname{Air}^{b}$ & $12.9 \pm 0.7 \mathrm{~B}$ & $9.6 \pm 0.8 \mathrm{~A}$ & $9.6 \pm 0.8 \mathrm{~A}$ & $10.1 \pm 1.2 * \mathrm{~A}$ \\
\hline & Nitrogen ${ }^{\mathrm{b}}$ & $13.2 \pm 0.9 \mathrm{C}$ & $10.8 \pm 0.8 \mathrm{~B}$ & $8.8 \pm 1.0 \mathrm{AB}$ & $7.1 \pm 0.6 * \mathrm{~A}$ \\
\hline \multirow[t]{2}{*}{$\mathrm{Y}_{\mathrm{E} / \mathrm{S}}(\mathrm{g} / \mathrm{g})$} & $\operatorname{Air}^{b}$ & $0.316 \pm 0.005 * \mathrm{~B}$ & $0.150 \pm 0.032 * \mathrm{~A}$ & $0.165 \pm 0.025 * \mathrm{~A}$ & $0.197 \pm 0.091 * \mathrm{AB}$ \\
\hline & Nitrogen & $0.399 \pm 0.001 *$ & $0.375 \pm 0.030^{*}$ & $0.362 \pm 0.043 *$ & $0.357 \pm 0.040^{*}$ \\
\hline \multirow[t]{2}{*}{$\mathrm{Y}_{\mathrm{A} / \mathrm{s}}(\mathrm{mg} / \mathrm{g})$} & $\operatorname{Air}^{\mathrm{b}}$ & $6.831 \pm 0.523 * \mathrm{~B}$ & $0.953 \pm 0.394 \mathrm{~A}$ & $0.883 \pm 0.241 \mathrm{~A}$ & $0.821 \pm 0.227 \mathrm{~A}$ \\
\hline & Nitrogen & $1.218 \pm 0.831^{*}$ & $0.592 \pm 0.116$ & $0.670 \pm 0.112$ & $0.641 \pm 0.207$ \\
\hline \multirow[t]{2}{*}{$\mathrm{Y}_{\mathrm{G} / \mathrm{S}}(\mathrm{g} / \mathrm{g})$} & $\operatorname{Air}^{\mathrm{b}}$ & $0.054 \pm 0.001 * \mathrm{~A}$ & $0.129 \pm 0.028 \mathrm{~B}$ & $0.127 \pm 0.014 \mathrm{~B}$ & $0.120 \pm 0.031 \mathrm{~B}$ \\
\hline & Nitrogen ${ }^{\mathrm{b}}$ & $0.070 \pm 0.001 * \mathrm{~A}$ & $0.098 \pm 0.010 \mathrm{AB}$ & $0.125 \pm 0.019 \mathrm{BC}$ & $0.148 \pm 0.014 \mathrm{C}$ \\
\hline
\end{tabular}

Values are shown as mean \pm standard deviation of three biological replicates.

$Y_{E / S}$ ethanol yield on sugar, $Y_{A / S}$ acetic acid yield on sugar, $Y_{G / S}$ glycerol yield on sugar

${ }^{a}$ Statistically significant differences (ANOVA) between cultures sparged with air or nitrogen for the same parameter and inoculum are indicated by *

${ }^{\mathrm{b}}$ Different capital letters indicate statistically significant differences (ANOVA) for values in the same row 
Table 2 Concentration and yields of the main fermentation metabolites by the end (262-265 g/L sugar consumed) of fermentations sparged with air or nitrogen in the conditions described in the text

\begin{tabular}{|c|c|c|c|c|}
\hline & & S. cerevisiae $^{\mathrm{a}}$ & M. pulcherrima $+10 \%$ S. cerevisiae $^{\mathrm{a}}$ & M. pulcherrima $+1 \%$ S. cerevisiae $^{\mathrm{a}}$ \\
\hline \multirow[t]{2}{*}{ Glycerol $(\% w / v)$} & $\mathrm{Air}^{\mathrm{b}}$ & $0.83 \pm 0.02 * \mathrm{~A}$ & $1.86 \pm 0.18 * \mathrm{~B}$ & $1.79 \pm 0.06 \mathrm{~B}$ \\
\hline & Nitrogen $^{\mathrm{b}}$ & $1.20 \pm 0.04 * \mathrm{~A}$ & $1.46 \pm 0.06^{*} \mathrm{~B}$ & $1.65 \pm 0.06 \mathrm{C}$ \\
\hline \multirow[t]{2}{*}{ Ethanol $(\% v / v)$} & Air $^{\mathrm{b}}$ & $12.9 \pm 0.2 * \mathrm{~B}$ & $11.0 \pm 0.3 * \mathrm{~A}$ & $11.1 \pm 0.2 * \mathrm{~A}$ \\
\hline & Nitrogen $^{\mathrm{b}}$ & $14.7 \pm 0.2 *$ & $13.9 \pm 0.6^{*}$ & $13.9 \pm 0.4 *$ \\
\hline \multirow[t]{2}{*}{ Acetic acid (mg/L) } & Air $^{\mathrm{b}}$ & $2158 \pm 329 * \mathrm{~B}$ & $676 \pm 63 * \mathrm{~A}$ & $682 \pm 123 * A$ \\
\hline & Nitrogen & $185 \pm 47 * \mathrm{~B}$ & $63 \pm 3 * \mathrm{~A}$ & $62 \pm 2 * \mathrm{~A}$ \\
\hline \multirow[t]{2}{*}{$\mathrm{Y}_{\mathrm{E} / \mathrm{S}}(\mathrm{g} / \mathrm{g})$} & Air $^{b}$ & $0.384 \pm 0.007 * \mathrm{~B}$ & $0.329 \pm 0.010 * \mathrm{~A}$ & $0.330 \pm 0.006 * \mathrm{~A}$ \\
\hline & Nitrogen $^{\mathrm{b}}$ & $0.441 \pm 0.006^{*}$ & $0.417 \pm 0.014 *$ & $0.416 \pm 0.010^{*}$ \\
\hline \multirow[t]{2}{*}{$\mathrm{Y}_{\mathrm{A} / \mathrm{s}}(\mathrm{mg} / \mathrm{g})$} & Air $^{b}$ & $8.159 \pm 1.241 * \mathrm{~B}$ & $2.553 \pm 0.237 * \mathrm{~A}$ & $2.579 \pm 0.461 * \mathrm{~A}$ \\
\hline & Nitrogen & $0.703 \pm 0.178 * \mathrm{~B}$ & $0.238 \pm 0.010 * \mathrm{~A}$ & $0.236 \pm 0.007 * \mathrm{~A}$ \\
\hline \multirow[t]{2}{*}{$\mathrm{Y}_{\mathrm{G} / \mathrm{S}}(\mathrm{g} / \mathrm{g})$} & Air $^{\mathrm{b}}$ & $0.031 \pm 0.001 * \mathrm{~A}$ & $0.070 \pm 0.007 * \mathrm{~B}$ & $0.067 \pm 0.002 \mathrm{~B}$ \\
\hline & Nitrogen $^{\mathrm{b}}$ & $0.045 \pm 0.001 * \mathrm{~A}$ & $0.055 \pm 0.002 * \mathrm{~B}$ & $0.063 \pm 0.002 \mathrm{C}$ \\
\hline
\end{tabular}

Values are shown as mean \pm standard deviation of three biological replicates

$\mathrm{Y}_{\mathrm{E} / \mathrm{S}}$ ethanol yield on sugar, $\mathrm{Y}_{\mathrm{A} / \mathrm{S}}$ acetic acid yield on sugar, $\mathrm{Y}_{\mathrm{G} / \mathrm{S}}$ glycerol yield on sugar

${ }^{a}$ Statistically significant differences (ANOVA) between cultures sparged with air or nitrogen for the same parameter and inoculum are indicated by *

${ }^{\mathrm{b}}$ Different capital letters indicate statistically significant differences (ANOVA) for values in the same row

The lowest ethanol production values were observed for aerated fermentations. Reduction was of about $1.8 \%(v / v)$ for S. cerevisiae and $2.8 \%(v / v)$ for mixed cultures (Table 2$)$, as compared to anaerobic fermentation. The alcohol level reduction obtained with aerated mixed cultures, compared to anaerobic fermentations with $S$. cerevisiae, was around $3.7 \%(v / v)$ (11.0-11.1 vs $14.7 \%$ ethanol, respectively) (Table 2).

Acetic acid production for mixed cultures was about one third that of S. cerevisiae, under either air or nitrogen sparging. Unfortunately, even for mixed cultures, the levels reached in aerated fermentations were above $0.65 \mathrm{~g} / \mathrm{L}$ (Table 2), which

Table 3 Anaerobic stage-specific yields calculated for the main fermentation metabolites after sparging was completely stopped (from day 2 to the end of fermentation)

\begin{tabular}{llll}
\hline & & S. cerevisiae $^{\mathrm{a}}$ & $\begin{array}{l}\text { M. pulcherrima } \\
+10 \% \text { S. cerevisiae }\end{array}$ \\
\hline $\mathrm{Y}_{\mathrm{G} / \mathrm{S}}(\mathrm{g} / \mathrm{g})$ & Air & $0.010 \pm 0.004^{\mathrm{b}} \mathrm{A}$ & $0.037 \pm 0.000^{*} \mathrm{~B}$ \\
& Nitrogen & $0.020 \pm 0.003^{*}$ & $0.026 \pm 0.006^{*}$ \\
$\mathrm{Y}_{\mathrm{E} / \mathrm{S}}(\mathrm{g} / \mathrm{g})$ & Air & $0.451 \pm 0.023$ & $0.431 \pm 0.024$ \\
& Nitrogen & $0.481 \pm 0.007$ & $0.446 \pm 0.022$ \\
$\mathrm{Y}_{\mathrm{A} / \mathrm{S}}(\mathrm{mg} / \mathrm{g})$ & Air & $9.513 \pm 2.796^{*} \mathrm{~B}$ & $3.454 \pm 0.519^{*} \mathrm{~A}$ \\
& Nitrogen & $0.125 \pm 0.619^{*}$ & $-0.010 \pm 0.089^{*}$ \\
\hline
\end{tabular}

Values are shown as mean \pm standard deviation of three biological replicates

$Y_{E / S}$ ethanol yield on sugar, $Y_{A / S}$ acetic acid yield on sugar, $Y_{G / S}$ glycerol yield on sugar

${ }^{a}$ Statistically significant differences (ANOVA) between cultures sparged with air or nitrogen for the same parameter and inoculum are indicated by *

${ }^{\mathrm{b}}$ Different capital letters indicate statistically significant differences (ANOVA) for values in the same row would not be acceptable for most consumers or market regulations in different countries. Finally, the trend toward increased glycerol yield by mixed, aerated fermentations, already observed for $48 \mathrm{~h}$ samples, was confirmed as statistically significant by the end of fermentation (Table 2).

Impact of different aeration levels

Two additional oxygenation levels were assayed with pure S. cerevisiae and mixed cultures ( $10 \%$ S. cerevisiae $)$. This was done by following the same gassing pattern as above but using gas mixtures containing 10 or $25 \%$ air, which would result in maximum dissolved oxygen levels around 0.7 and $1.7 \mathrm{mg} / \mathrm{L}$ respectively. Data on main fermentation metabolites for these experiments are shown in Table 4. Considering data from the four oxygenation levels, a negative correlation between air concentration and ethanol yield was found for both inocula (Table 5). In addition, for each condition, ethanol yield was always lower for the mixed culture than for $S$. cerevisiae (Tables 2 and 4).

Positive correlations were confirmed between acetic acid yield and oxygenation level (Table 5). However, glycerol yields showed opposite trends when considering the pure $S$. cerevisiae cultures or the mixed ones. In addition, while the trend for $S$. cerevisiae pure cultures is clear, a J-shaped graph was obtained for the mixed cultures (not shown).

Population dynamics

Under all experimental conditions, cell growth took place mainly during the first $48 \mathrm{~h}$ of culture (Fig. 1). The highest 
Table 4 Concentration and yields of the main fermentation metabolites by the end of fermentations sparged with air/nitrogen mixtures in the conditions described in the text

\begin{tabular}{llll}
\hline & \multicolumn{3}{c}{ Inoculum } \\
\cline { 3 - 4 } & & S. cerevisiae & $\begin{array}{c}\text { M. pulcherrima }+ \\
10 \% \text { S.cerevisiae }\end{array}$ \\
\hline Glycerol $(\% w / v)$ & $10 \%$ Air $^{\mathrm{a}}$ & $1.06 \pm 0.05 \mathrm{~A}$ & $1.21 \pm 0.01 \mathrm{~B}$ \\
& $25 \% \mathrm{Air}^{\mathrm{a}}$ & $0.97 \pm 0.02 \mathrm{~A}$ & $1.11 \pm 0.01 \mathrm{~B}$ \\
Ethanol $(\% v / v)$ & $10 \% \mathrm{Air}^{\mathrm{a}}$ & $14.2 \pm 0.0 \mathrm{~B}$ & $13.2 \pm 0.1 \mathrm{~A}$ \\
& $25 \% \mathrm{Air}^{\mathrm{a}}$ & $13.8 \pm 0.1 \mathrm{~B}$ & $12.6 \pm 0.2 \mathrm{~A}$ \\
Acetic acid $(\mathrm{mg} / \mathrm{L})$ & $10 \% \mathrm{Air}^{\mathrm{a}}$ & $451 \pm 71 \mathrm{~B}$ & $208 \pm 3 \mathrm{~A}$ \\
& $25 \% \mathrm{Air}^{\mathrm{a}}$ & $1074 \pm 267$ & $351 \pm 15$ \\
$\mathrm{Y}_{\mathrm{E} / \mathrm{S}}(\mathrm{g} / \mathrm{g})$ & $10 \% \mathrm{Air}^{\mathrm{a}}$ & $0.425 \pm 0.001 \mathrm{~B}$ & $0.394 \pm 0.002 \mathrm{~A}$ \\
& $25 \% \mathrm{Air}^{\mathrm{a}}$ & $0.412 \pm 0.003 \mathrm{~B}$ & $0.377 \pm 0.005 \mathrm{~A}$ \\
$\mathrm{Y}_{\mathrm{A} / \mathrm{S}}(\mathrm{mg} / \mathrm{g})$ & $10 \% \mathrm{Air}^{\mathrm{a}}$ & $1.706 \pm 0.268 \mathrm{~B}$ & $0.787 \pm 0.011 \mathrm{~A}$ \\
& $25 \% \mathrm{Air}^{\mathrm{a}}$ & $4.060 \pm 1.008$ & $1.326 \pm 0.055$ \\
$\mathrm{Y}_{\mathrm{G} / \mathrm{S}}(\mathrm{g} / \mathrm{g})$ & $10 \% \mathrm{Air}^{\mathrm{a}}$ & $0.040 \pm 0.002 \mathrm{~A}$ & $0.046 \pm 0.000 \mathrm{~B}$ \\
& $25 \% \mathrm{Air}$ & $0.036 \pm 0.001 \mathrm{~A}$ & $0.042 \pm 0.001 \mathrm{~B}$ \\
\hline
\end{tabular}

Values are shown as mean \pm standard deviation of two biological replicates

$Y_{E / S}$ ethanol yield on sugar, $Y_{A / S}$ acetic acid yield on sugar, $Y_{G / S}$ glycerol yield on sugar

${ }^{a}$ Different capital letters indicate statistically significant differences for values in the same row $(t$ test $)$

biomass values were obtained in fermentation experiments sparged with air and inoculated with M. pulcherrima either alone or in mixed culture, reaching $\mathrm{OD}_{600}$ values close to 50 (Fig. 1). Under these conditions, $\mathrm{OD}_{600}$ values for $S$. cerevisiae pure cultures were about half those of pure M. pulcherrima. Cultures sparged with nitrogen reached much lower biomass values, as expected for pure fermentative metabolism. In addition, the relative advantages of $S$. cerevisiae and $M$. pulcherrima were inverted when cultures were sparged with nitrogen. Under these conditions, mixed and pure $S$. cerevisiae cultures reached $\mathrm{OD}_{600}$ values clearly higher than the M. pulcherrima pure culture (Fig. 1). In cultures sparged with air/nitrogen mixtures (10 or $25 \%$ air), biomass

Table 5 Correlation between the final yields of main fermentation metabolites and air concentration in the sparging gas for different inocula

\begin{tabular}{lcc}
\hline & S.cerevisiae & M.pulcherrima $+10 \%$ S.cerevisiae \\
\hline $\mathrm{Y}_{\mathrm{E} / \mathrm{S}}$ & $-0.947^{* *}$ & $-0.953^{* *}$ \\
$\mathrm{Y}_{\mathrm{A} / \mathrm{S}}$ & $0.962^{* *}$ & $0.970^{* *}$ \\
$\mathrm{Y}_{\mathrm{G} / \mathrm{S}}$ & $-0.908^{* *}$ & $0.754^{*}$ \\
\hline
\end{tabular}

$Y_{E / S}$ ethanol yield on sugar, $Y_{A / S}$ acetic acid yield on sugar, $Y_{G / S}$ glycerol yield on sugar

*Statistically significant at the 0.05 level

**Statistically significant at the 0.01 level
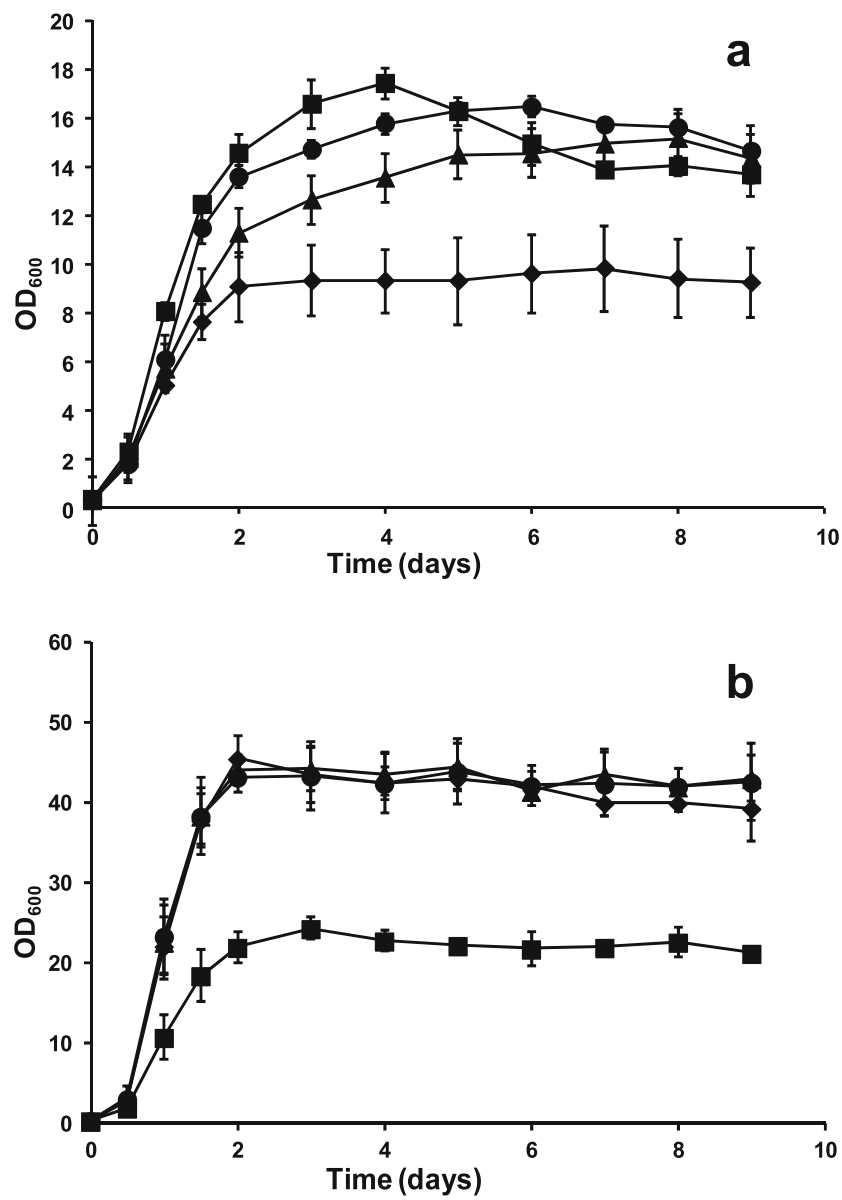

Fig. 1 Cell growth in fermentations sparged with nitrogen (a) or air (b), in the conditions described in the text, for different combinations of yeast strains in the inoculums: pure $M$. pulcherrima (diamonds), pure $S$. cerevisiae (squares), M. pulcherrima plus $1 \%$ S. cerevisiae (triangles), and M. pulcherrima plus $10 \%$ S. cerevisiae (circles). Note that vertical axes are different between panels

production was closer to cultures sparged with air than to anaerobic cultures (data not shown). These results are in agreement with our previous demonstration of respiratory metabolism for $S$. cerevisiae EC1118 and M. pulcherrima CECT12841 under similar experimental conditions (Quirós et al. 2014).

In mixed cultures, M. pulcherrima was always replaced by $S$. cerevisiae by the end of the fermentation (Fig. 2). This replacement took place later for cultures sparged with air (around day 7) than for those sparged with nitrogen (around day 4). Indeed, growth of M. pulcherrima was dramatically restricted under nitrogen sparging, even in pure culture (Fig. 1). For oxygenated cultures, decay of this species was slower with increasing proportion of air during the gassing step, despite having reached similar maximum viable cell numbers. Growth of $S$. cerevisiae in these mixed cultures was very similar for all gassing conditions (Fig. 2). Maximum colony forming unit (CFU) counts fell below those of M. pulcherrima, except for the most anaerobic condition, 
but remained constant until the end of the fermentation process, in contrast to the relatively quick decrease in M. pulcherrima viability (Fig. 2). Viable counts for pure $S$. cerevisiae cultures were generally higher than in mixed cultures (Fig. 2).

\section{Dissolved oxygen}

We monitored DO levels during fermentation experiments as an indicator of the potential risk of oxidation of grape must components. DO levels during the first $48 \mathrm{~h}$ were absolutely dependent of the composition of the gas used to sparge the culture but also strongly dependent on the strain used. For all
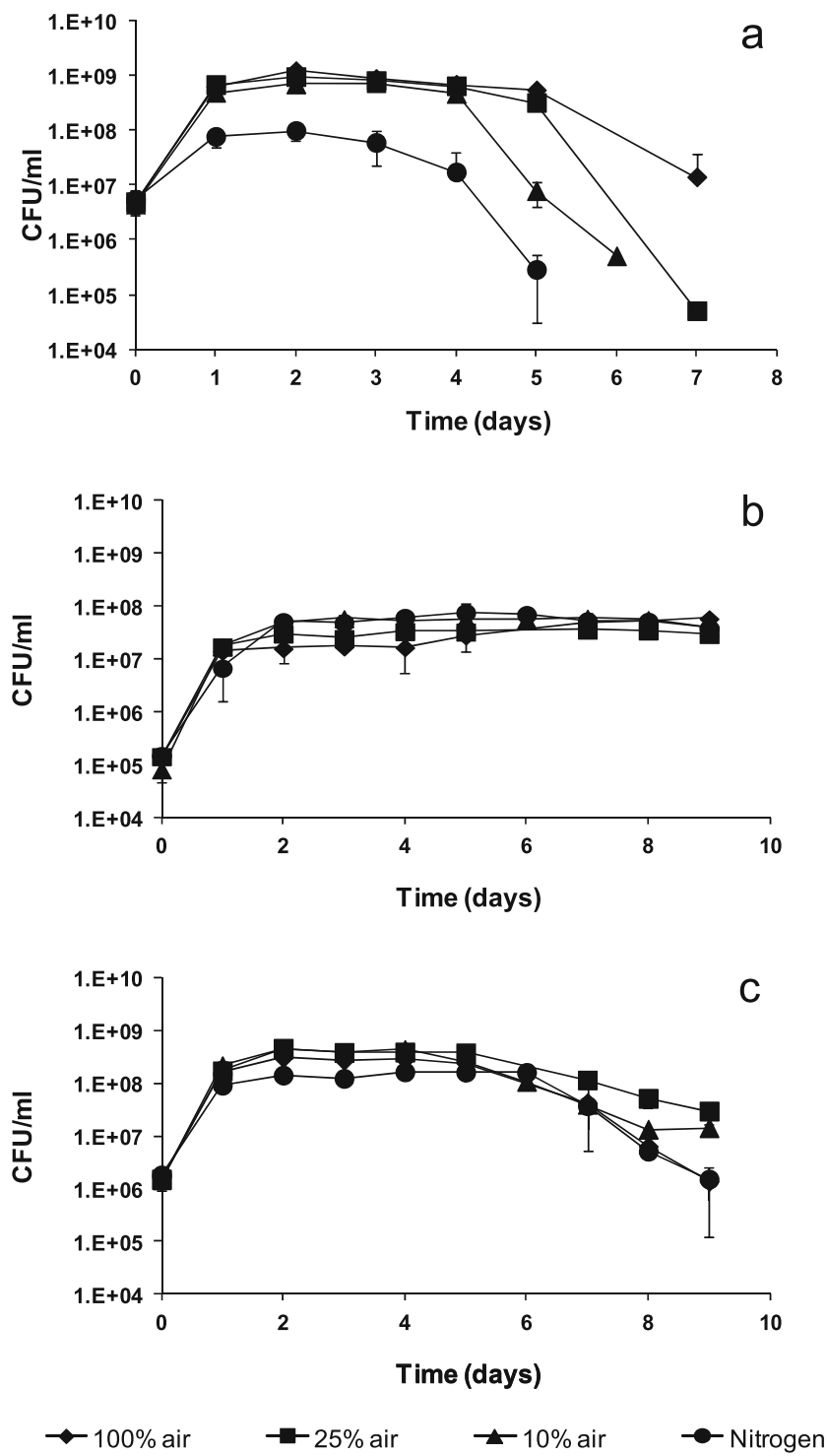

Fig. 2 Viable cell counts for fermentations sparged with different gas mixtures, in the conditions described in the text, for a M. pulcherrima in cultures inoculated with $M$. pulcherrim $+10 \%$ S. cerevisiae; b $S$. cerevisiae in cultures inoculated with $M$. pulcherrima $+10 \%$ $S$. cerevisiae; or c $S$. cerevisiae pure cultures cultures inoculated with $M$. pulcherrima (either pure or in combination with $S$. cerevisiae), DO gradually dropped and stabilized around zero, even between 24 and $40 \mathrm{~h}$, when sparging was continuous (Fig. 3). In contrast, cultures inoculated with $S$. cerevisiae alone showed DO levels above $50 \%$ (i.e. about $3.5 \mathrm{mg} / \mathrm{L}$ ) for most of the time; apart from the short periods, sparging was stopped, especially from 40 to $48 \mathrm{~h}$ (Fig. 3). As expected, DO values were clearly lower when $25 \%$ instead of $100 \%$ air was used (Fig. 3). Basal level was reached faster than in experiments performed with $100 \%$ air and quicker for the mixed culture than for $S$. cerevisiae alone (Fig. 3). Not surprisingly, DO levels dropped to zero immediately after sparging was stopped, for any strain combination or gas composition.

\section{Discussion}

Growth

In agreement with our previous observations, $M$. pulcherrima showed better growth and sugar consumption under aerobic conditions (Table 1 and Fig. 1), as compared to anaerobiosis. However, after the switch to anaerobic conditions, the M. pulcherrima pure cultures were not able to complete fermentation. Slow or incomplete fermentations are common for non-Saccharomyces yeast strains and have been previously described for M. pulcherrima (Medina et al. 2012), although Sadoudi et al. (2012) recently described complete grape must fermentation by one strain of this species.

Under anaerobic conditions, faster fermentative metabolism confers a growth advantage to $S$. cerevisiae over M. pulcherrima (Fig. 1). However, M. pulcherrima is able to take better advantage of oxygen when it is available (Fig. 1). In mixed cultures, changes in the initial aeration regime had a stronger impact on the growth of M. pulcherrima than on S. cerevisiae (Fig. 2), resulting in a quick yeast strain replacement under anaerobic conditions. A competitive disadvantage for other non-Saccharomyces species in the absence of oxygen was previously described by Hansen et al. (2001). However, other mechanisms might also be involved in yeast-yeast competitive interactions, as shown by Nissen et al. (2003) or Bely et al. (2008) for different S. cerevisiael Torulaspora delbrueckii strain combinations.

\section{Ethanol}

Both M. pulcherrima and S. cerevisiae showed reduced ethanol yield in aerobic as compared to anaerobic cultures (Table 1). However, as expected for Crabtree-negative yeasts (Quirós et al. 2014), the extent of this reduction was higher for M. pulcherrima. The sudden removal of the air supply after 

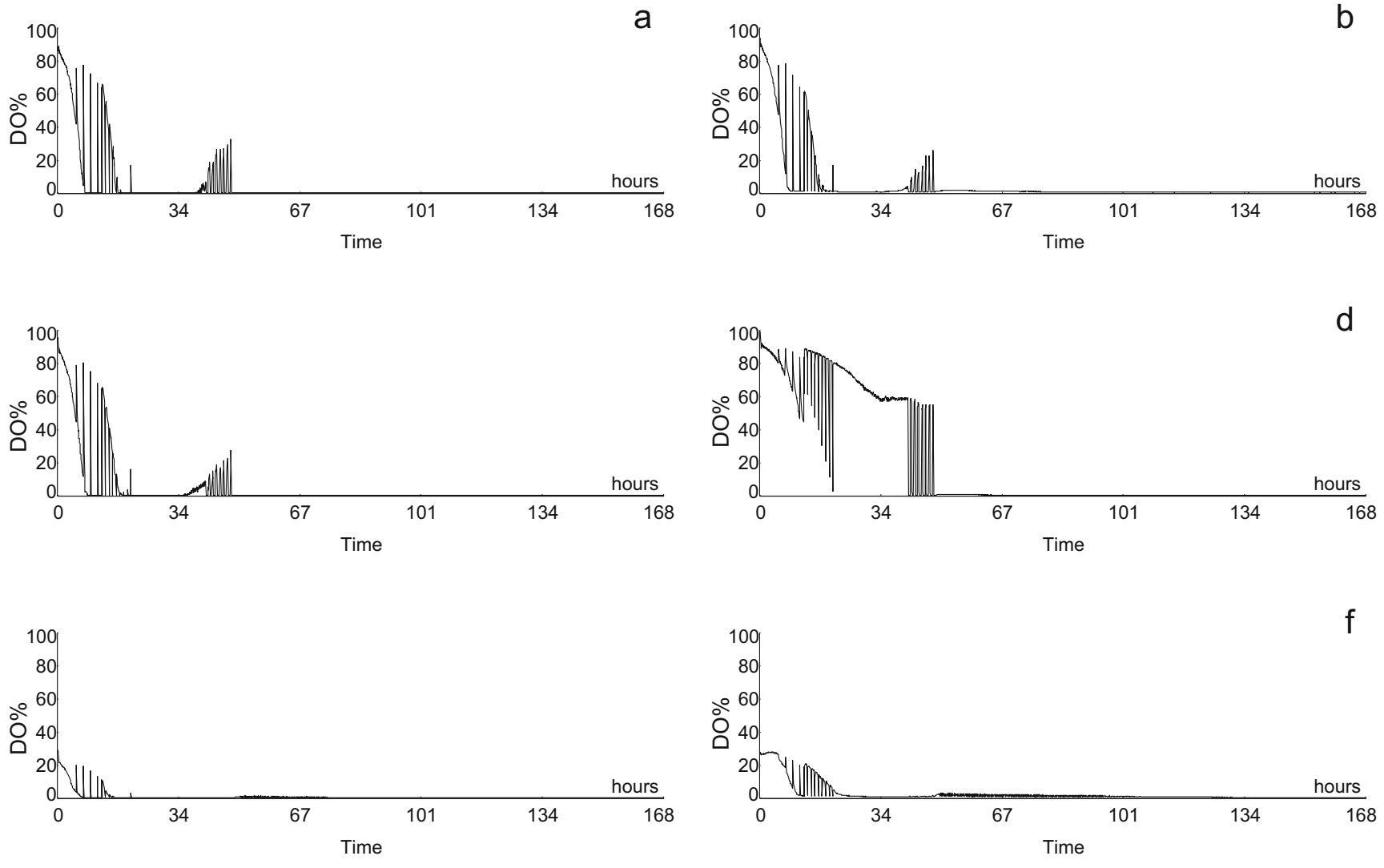

Fig. 3 Evolution of dissolved oxygen levels (expressed as \% saturation with air) in fermentations sparged with $100 \%$ air $(\mathbf{a}-\mathbf{d})$ or $25 \%$ air $(\mathbf{e}-\mathbf{f})$ in the conditions described in the text for different combinations of yeast

strains in the inoculum: M. pulcherrima $(\mathbf{a}) ; S$. cerevisiae $(\mathbf{d}, \mathbf{f})$; M. pulcherrima $+10 \%$ S. cerevisiae (c, e); M. pulcherrima $+1 \%$ S. cerevisiae (b)

$48 \mathrm{~h}$ resulted in increasing ethanol yields in the second part of the experiments for all culture conditions (Table 3). In agreement with our working hypothesis, as well as results by Giovanelli et al. (1996), a strong and significantly negative correlation between air concentration and the final yield of ethanol was found for both S. cerevisiae and mixed cultures (Table 5). Differences in final alcohol content between fully anaerobic or air sparged cultures were already relevant for pure $S$. cerevisiae cultures, $2.2 \%(v / v)$, but were more important for mixed cultures, $2.9 \%(v / v)$ and up to $3.7 \%(v / v)$, when comparing anaerobic $S$. cerevisiae against aerated mixed cultures (Table 2). In addition to the shift from respirofermentative to pure fermentative metabolism, gradual replacement of $M$. pulcherrima by $S$. cerevisiae must also be contributing to the increase in final ethanol yield observed for mixed cultures in the second part of the experiments.

Contreras et al. (2014) described alcohol level reduction in non-aerated sequential inoculation cultures with one strain of M. pulcherrima and one of $S$. cerevisiae. This would be in agreement with the trend observed in Table 2 for nitrogensparged cultures. Other authors had also reported moderate alcohol level reductions by using non-Saccharomyces yeast strains, either pure or in co-culture, under non-aerated

conditions (Ciani and Comitini 2006; Bely et al. 2008; Gobbi et al. 2013). Understanding the fate of carbon in those cases would require further analysis and improved knowledge on the metabolism of these non-conventional yeasts.

Acetic acid and glycerol

The most noticeable impact of aeration on $S$. cerevisiae metabolism was on acetic acid production (Table 1). Moreover, a positive correlation was confirmed between acetic acid yield and oxygenation level (Table 5). This is in agreement with results by Giovanelli et al. (1996) who also found increased acetic acid yield under aerobic conditions (as compared to anaerobic) for the fermentation of commercial grape must. Likewise, Franzén (2003) found increased acetic acid yields with decreasing RQ values for this species. All these results are in contrast to those by Aceituno et al. (2012) who described acetic acid production to take place only under fully anaerobic conditions. Probably, the use of nitrogen-limited chemostat by Aceituno et al. (2012) is related to the differences they found in the pattern of acetic acid production, as compared to other authors. 
Prior differences in the aeration regime had a huge impact on stage-specific acetic acid yields, even after sparging was completely stopped (Table 3 ). This can be related to differences in the biomass content or in the metabolic features of the cells, depending on the environmental conditions during the growth phase. Short-term aeration practices had been previously reported to influence global yeast physiology and fermentation kinetics (Valero et al. 2001; Fornairon-Bonnefond et al. 2003; Varela et al. 2012b). Interestingly, the use of mixed cultures conferred a clear advantage over pure $S$. cerevisiae to control volatile acidity, especially under intermediate oxygenation levels (Tables 2 and 4).

Dependence of glycerol yields on oxygen availability showed opposite trends for pure S. cerevisiae (negative correlation) or mixed cultures (positive correlation). The J-shaped graph obtained for mixed cultures seems to result from the differential effect of oxygen availability on the yeast strains used. By one side, according to data by Giovanelli et al. (1996) and results shown in Table 1, S. cerevisiae shows a greater glycerol yield under nitrogen sparging, being the most active strain under these conditions. By the other side, M. pulcherrima survival is favored under air sparging while it shows a higher glycerol yield than $S$. cerevisiae under all conditions (Table 1). Under intermediate oxygenation conditions, $S$. cerevisiae will tend to lower glycerol yield, while M. pulcherrima will not be so favored, resulting in lower glycerol yields than the extreme conditions. Metabolic interactions between the strains might also influence the different yields, as described for other $S$. cerevisiae/nonSaccharomyces strain combinations (Sadoudi et al. 2012; Milanovic et al. 2012).

\section{Dissolved oxygen}

Oxygen consumption by $M$. pulcherrima in mixed cultures led to low DO levels during most of the aeration step, in contrast to pure S. cerevisiae cultures (Fig. 3). The use of air mixtures further helped to reduce DO levels. In all cases, DO fell to zero in the second, anaerobic step, as intended. Oxygen affinity of wine polyhenols has been determined to be about 1000 times lower than fermenting S. cerevisiae cells (Salmon 2006), so according to data shown in Fig. 3, we can expect a very low impact of the aeration regime described in this work on the oxidation of grape must components for fermentations driven by M. pulcherrima CECT12841 (alone or including up to $10 \%$ of $S$. cerevisiae EC1118).

In summary, both the use of M. pulcherrima CECT12841 and air sparging during the first $48 \mathrm{~h}$ have a great impact on fermentation dynamics and the production of yeast metabolites during growth in natural grape must. Most of the observed effects could be explained in terms of differences in central carbon metabolism between the two yeast strains employed, either directly or through its influence on population dynamics. This work shows the potential of sugar respiration by non-Saccharomyces yeasts to help reduce alcohol levels in wine, as previously suggested (Gonzalez et al. 2013). More recently, Contreras et al. (2014) described decreased ethanol yields by sequential inoculation of $M$. pulcherrima and S. cerevisiae. Using different strains of both species and simultaneous inoculation, we showed the crucial role of oxygen availability and respiratory metabolism in order to reduce alcohol levels by up to $3.7 \%(v / v)$ by the end of fermentation of a natural white grape must. By choosing and optimizing the appropriate gassing conditions (i.e. $25 \%$ air), we managed to find a good balance between alcohol level reduction $(2.2 \%(v / v))$, the increase in volatile acidity, mostly associated to growth of $S$. cerevisiae under aerobic conditions (below $0.35 \mathrm{~g} / \mathrm{L}$ ), and levels of dissolved oxygen during the process (most time being almost undetectable). However, we should keep in mind that practical constraints will be different between the industrial and the laboratory setup. For example, intermediate aeration levels were attained in this work by using gas mixtures (for operational reasons), but they could be reached in an industrial setup by simply reducing the gas flow and avoiding agitation. Therefore, the implementation at the industrial level of a strategy to lower ethanol content of wine, based on the respiratory breakdown of sugars by non-Saccharomyces yeasts, poses an interesting challenge that would require further optimization, involving yeast species and strain selection, inoculation strategies, development of oxygenation and mixing conditions and devices, or fermentation nutrients.

Acknowledgments Authors would like to thank Cristina Juez Ojeda and Miguel Ángel Fernández Recio for excellent technical assistance, the YeSVitE consortium (EU project, 7FP-IRSES-GA no. 612441) for helpful discussions, and Laura López Ocaña (CECT) for help with yeast species confirmation. This work was supported by grants MINECO AGL2012-32064 and INIA RM2012-00007-00-00 from the Spanish Government. M.Q. was the recipient of a CSIC training contract, JAEDoc, co-funded by the European Social Fund of the EU.

Open Access This article is distributed under the terms of the Creative Commons Attribution License which permits any use, distribution, and reproduction in any medium, provided the original author(s) and the source are credited.

\section{References}

Aceituno FF, Orellana M, Torres J, Mendoza S, Slater AW, Melo F, Agosin E (2012) Oxygen response of the wine yeast Saccharomyces cerevisiae EC1118 grown under carbon-sufficient, nitrogen-limited enological conditions. Appl Environ Microbiol 78: $8340-8352$ 
Bärwald G, Fischer A (1996) Crabtree effect in aerobic fermentations using grape juice for the production of alcohol reduced wine. Biotechnol Lett 18:1187-1192

Belisario-Sánchez YY, Taboada-Rodríguez A, Marín-Iniesta F, LópezGómez A (2009) Dealcoholized wines by spinning cone column distillation: phenolic compounds and antioxidant activity measured by the 1,1-diphenyl-2-picrylhydrazyl method. J Agric Food Chem 57:6770-6778

Bely M, Stoeckle P, Masneuf-Pomarede I, Dubourdieu D (2008) Impact of mixed Torulaspora delbrueckii-Saccharomcyes cerevisiae culture on high-sugar fermentation. Int J Food Microbiol 122:312-320

Cadiere A, Ortiz-Julien A, Camarasa C, Dequin S (2011) Evolutionary engineered Saccharomyces cerevisiae wine yeast strains with increased in vivo flux through the pentose phosphate pathway. Metab Eng 13:263-271

Canals R, Llaudy MDC, Canals JM, Zamora F (2008) Influence of the elimination and addition of seeds on the colour, phenolic composition and astringency of red wine. Eur Food Res Technol 226:11831190

Catarino M, Mendes A (2011) Dealcoholizing wine by membrane separation processes. Inn Food Sci Emerg Technol 12:330-337

Ciani M, Comitini F (2006) Influence of temperature and oxygen concentration on the fermentation behaviour of Candida stellata in mixed fermentation with Saccharomyces cerevisiae. World J Microbiol Biotechnol 22:619-623

Ciani M, Comitini F, Mannazzu I, Domizio P (2010) Controlled mixed culture fermentation: a new perspective on the use of nonSaccharomyces yeasts in winemaking. FEMS Yeast Res 10:123133

Contreras A, Hidalgo C, Henschke PA, Chambers PJ, Curtin C, Varela C (2014) Evaluation of non-Saccharomyces yeast for the reduction of alcohol content in wine. Appl Environ Microbiol 80:1670-1678

Cordero-Bueso G, Esteve-Zarzoso B, Cabellos JM, Gil-Díaz M, Arroyo T (2013) Biotechnological potential of non-Saccharomyces yeasts isolated during spontaneous fermentations of Malvar (Vitis vinifera cv. L.). Eur Food Res Technol 236:193-207

Dukes BC, Butzke CE (1998) Rapid determination of primary amino acids in grape juice using an $o$-phthaldialdehyde/ $\mathrm{N}$-acetyl-L-cysteine spectrophotometric assay. Am J Enol Vitic 49:125-134

Erten H, Campbell I (2001) The production of low-alcohol wines by aerobic yeasts. J Inst Brew 107:207-215

Fleet GH (2003) Yeast interactions and wine flavour. Int J Food Microbiol $86: 11-22$

Fleet GH (2007) Yeasts in foods and beverages: impact on product quality and safety. Curr Opin Biotechnol 18:170-175

Fornairon-Bonnefond C, Aguera E, Deyteieux C, Sablayrolles JM, Salmon JM (2003) Impact of oxygen addition during enological fermentation on sterol contents in yeast lees and their reactivity towards oxygen. J Biosci Bioeng 95:496-503

Franzén CJ (2003) Metabolic flux analysis of RQ-controlled microaerobic ethanol production by Saccharomyces cerevisiae. Yeast 20:117-132

Giovanelli G, Peri C, Parravicini E (1996) Kinetics of grape juice fermentation under aerobic and anaerobic conditions. Am J Enol Vitic 47:429-434

Gobbi M, Comitini F, Domizio P, Romani C, Lencioni L, Mannazzu I, Ciani M (2013) Lachancea thermotolerans and Saccharomyces cerevisiae in simultaneous and sequential co-fermentation: a strategy to enhance acidity and improve the overall quality of wine. Food Microbiol 33:271-281

Gonzalez R, Quirós M, Morales P (2013) Yeast respiration of sugars by non-Saccharomyces yeast species: a promising and barely explored approach to lowering alcohol content of wines. Trends Food Sci Technol 29:55-61

Hansen EH, Nissen P, Sommer P, Nielsen JC, Arneborg N (2001) The effect of oxygen on survival of non-Saccharomyces yeasts during mixed culture fermentations of grape juice with Saccharomyces cerevisiae. J Appl Microbiol 91:541-547

Henricsson C, de Jesus Ferreira MC, Hedfalk K, Elbing K, Larsson C, Bill RM, Norbeck J, Hohmann S, Gustafsson L (2005) Engineering of a novel Saccharomyces cerevisiae wine strain with a respiratory phenotype at high external glucose concentrations. Appl Environ Microbiol 71:6185-6192

Heux S, Sablayrolles JM, Cachon R, Dequin S (2006) Engineering a Saccharomyces cerevisiae wine yeast that exhibits reduced ethanol production during fermentation under controlled microoxygenation conditions. Appl Environ Microbiol 72:5822-5828

Kontoudakis N, Esteruelas M, Fort F, Canals JM, Zamora F (2011) Use of unripe grapes harvested during cluster thinning as a method for reducing alcohol content and $\mathrm{pH}$ of wine. Aust J Grape Wine Res 17:230-238

Kutyna DR, Varela C, Henschke PA, Chambers PJ, Stanley GA (2010) Microbiological approaches to lowering ethanol concentration in wine. Trends Food Sci Technol 21:293-302

Medina K, Boido E, Dellacassa E, Carrau F (2012) Growth of nonSaccharomyces yeasts affects nutrient availability for Saccharomyces cerevisiae during wine fermentation. Int J Food Microbiol 157:245-250

Medina K, Boido E, L. F, Gioia O, Gomez ME, Barquet M, Gaggero C, Dellacassa E, Carrau F (2013) Increased flavour diversity of Chardonnay wines by spontaneous fermentation and cofermentation with Hanseniaspora vineae. Food Chem 141:25132521

Michnick S, Roustan JL, Remize F, Barre P, Dequin S (1997) Modulation of glycerol and ethanol yields during alcoholic fermentation in Saccharomyces cerevisiae strains overexpressed or disrupted for GPD1 encoding glycerol 3-phosphate dehydrogenase. Yeast 13: 783-793

Milanovic V, Ciani M, Oro L, Comitini F (2012) Starmerella bombicola influences the metabolism of Saccharomyces cerevisiae at pyruvate decarboxylase and alcohol dehydrogenase level during mixed wine fermentation. Microb Cell Factories 11:18

Mira de Orduña R (2010) Climate change associated effects on grape and wine quality and production. Food Res Int 43:1844-1855

Nissen P, Nielsen D, Arneborg N (2003) Viable Saccharomyces cerevisiae cells at high concentrations cause early growth arrest of non-Saccharomyces yeasts in mixed cultures by a cell-cell contactmediated mechanism. Yeast 20:331-341

Quirós M, Martínez-Moreno R, Albiol J, Morales P, Vázquez-Lima F, Barreiro-Vázquez A, Ferrer P, Gonzalez R (2013) Metabolic flux analysis during the exponential growth phase of Saccharomyces cerevisiae in wine fermentations. PLoS One 8:e71909

Quirós M, Rojas V, Gonzalez R, Morales P (2014) Selection of nonSaccharomcyes yeast strains for reducing alcohol levels in wine by sugar respiration. Int J Food Microbiol 181:85-91

Rojas V, Gil JV, Pinaga F, Manzanares P (2003) Acetate ester formation in wine by mixed cultures in laboratory fermentations. Int $\mathrm{J}$ Food Microbiol 86:181-188

Rossouw D, Heyns EH, Setati ME, Bosch S, Bauer FF (2013) Adjustment of trehalose metabolism in wine Saccharomyces cerevisiae strains to modify ethanol yields. Appl Environ Microbiol 79:5197-5207

Saa PA, Moenne MI, Pérez-Correa JR, Agosin E (2012) Modeling oxygen dissolution and biological uptake during pulse oxygen additions in oenological fermentations. Bioprocess Biosyst Eng 35: $1167-1178$

Sadoudi M, Tourdot-Maréchal R, Rousseaux S, Steyer D, GallardoChacón JJ, Ballester J, Vichi S, Guérin-Schneider R, Caixach J, Alexandre H (2012) Yeast-yeast interactions revealed by aromatic profile analysis of Sauvignon Blanc wine fermented by single or coculture of non-Saccharomyces and Saccharomyces yeasts. Food Microbiol 32:243-253 
Salmon J-M (2006) Interactions between yeast, oxygen and polyphenols during alcoholic fermentations: practical implications. LWT Food Sci Technol 39:959-965

Schmidtke LM, Blackman JW, Agboola SO (2012) Production technologies for reduced alcoholic wines. J Food Sci 77:R25-R41

Smith PM (1995) Biological processes for the reduction of alcohol in wines. Master's Thesis. Lincoln University, Lincoln

Tamang JP, Fleet GH (2009) Yeasts diversity in fermented foods and beverages. In: Satyanarayana T, Kunze G (eds) Yeast biotechnology: diversity and applications. Springer Science+Business Media B.V, Dordrecht, pp 169-198

Tilloy V, Ortiz-Julien A, Dequin S (2014) Reduction of ethanol yield and improvement of glycerol formation by adaptive evolution of the wine yeast Saccharomyces cerevisiae under hyperosmotic conditions. Appl Environ Microbiol 80:2623-2632
Valero E, Millan C, Ortega JM (2001) Influence of oxygen addition during growth phase on the biosynthesis of lipids in Saccharomyces cerevisiae $\left(\mathrm{M}_{3} 30-9\right)$ in enological fermentations. J Biosci Bioeng 92:33-38

Varela C, Kutyna DR, Solomon MR, Black CA, Borneman A, Henschke PA, Pretorius IS, Chambers PJ (2012a) Evaluation of gene modification strategies for the development of low-alcohol-wine yeasts. Appl Environ Microbiol 78:6068-6077

Varela C, Pizarro F, Agosin E (2004) Biomass content governs fermentation rate in nitrogen-deficient wine musts. Appl Environ Microbiol 70:3392-3400

Varela C, Torrea D, Schmidt SA, Ancin-Azpilicueta C, Henschke PA (2012b) Effect of oxygen and lipid supplementation on the volatile composition of chemically defined medium and Chardonay wine fermented with Saccharomyces cerevisiae. Food Chem 135:2863-2871 